\title{
PT-Symmetry and Related Geometrical Structures ${ }^{\dagger}$
}

\author{
Uwe Günther \\ Helmholtz-Center Dresden-Rossendorf, 01328 Dresden, Germany; u.guenther@hzdr.de \\ + Presented at Symmetry 2017-The First International Conference on Symmetry, Barcelona, Spain, \\ 16-18 October 2017.
}

Published: 3 January 2018

\begin{abstract}
In non-relativistic quantum mechanics, the dynamics of closed quantum systems is described by Hamiltonians which are self-adjoint in appropriately chosen Hilbert spaces. For PTsymmetric quantum systems, the Hamiltonians are, in general, no longer self-adjoint in standard Hilbert spaces, rather they are self-adjoint in Krein spaces-Hilbert spaces endowed with indefinite metric structures. Moreover, the spectra of PT-symmetric Hamiltonians are symmetric with regard to the real axis in the spectral plane. Apart from Hamiltonians with purely real spectra, this includes also Hamiltonians whose spectra may contain sectors of pairwise complex-conjugate eigenvalues. Considering families of parameter-dependent Hamiltonians, one can arrange parameter-induced passages from sectors of purely real spectra to sectors of complex-conjugate spectral branches. Corresponding passages can be regarded as PT-phase transitions from sectors of exact PT-symmetry to sectors of spontaneously broken PT-symmetry. Approaching a PT-phase transition point, the eigenvectors of the Hamiltonian tend toward their isotropic limit-an, in general, infinitedimensional (Krein-space) generalization of the light-cone limit in Minkowski space. At a phase transition, the Hamiltonian is no longer diagonalizable, but similar to an arrangement of nontrivial Jordan-blocks. The interplay of these structures is briefly reviewed with special emphasis on the related Lie-algebraic and Lie-group aspects. With the help of Cartan-decompositions, associated hyperbolic structures and Lie-triple-systems are discussed for finite-dimensional setups as well as for their infinite-dimensional generalizations (Hilbert-Schmidt (HS) Lie groups, HS Lie algebras, HS Grassmannians). The interconnection of Krein-space structures and PT-phase transitions is demonstrated on two exactly solvable models: PT-symmetric Bose-Hubbard models and PTsymmetric plaquette arrangements.
\end{abstract}

(C) 2018 by the author. Licensee MDPI, Basel, Switzerland. This article is an open access article distributed under the terms and conditions of the Creative Commons Attribution (CC BY) license (http://creativecommons.org/licenses/by/4.0/). 